\section{PSICOLOGIA IBEROAMERICANA}

\section{Psicología lberoamericana}

ISSN: 1405-0943

psicología.iberoamericana@uia.mx

Universidad Iberoamericana, Ciudad de

México

México

Valdez Medina, José Luis; Álvarez González, Arely Marlen; González Gómez Tagle, Diana; González

Arratia López Fuentes, Norma Ivonne; González Escobar, Sergio Tipos de Miedo más Frecuentes en Niños de Primaria: Un Análisis por Sexo

Psicología Iberoamericana, vol. 18, núm. 1, enero-junio, 2010, pp. 47-55

Universidad Iberoamericana, Ciudad de México

Distrito Federal, México

Disponible en: http://www.redalyc.org/articulo.oa?id=133915936006

- Cómo citar el artículo

Número completo

- Más información del artículo

Página de la revista en redalyc.org

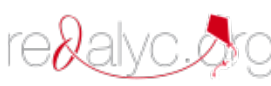

Sistema de Información Científica

Red de Revistas Científicas de América Latina, el Caribe, España y Portugal Proyecto académico sin fines de lucro, desarrollado bajo la iniciativa de acceso abierto 


\title{
Tipos de Miedo más Frecuentes en Niños de Primaria: Un Análisis por Sexo
}

\author{
Most Frequent Types of Fear in Children of Primary: An Analysis by Sex
}

\author{
José Luis Valdez Medina \\ Arely Marlen Álvarez González \\ Diana González Gómez Tagle \\ Norma Ivonne González Arratia López Fuentes \\ Sergio González Escobar * \\ Universidad Autónoma del Estado de México, México
}

\section{RESUMEN}

Con el objetivo de identificar los principales tipos de miedos que se presentan en los niños de primaria, se evaluaron 300 participantes de la ciudad de Toluca, de quinto y sexto grado, repartidos equitativamente por sexo. Para obtener la información requerida, se trabajó con el instrumento "Tipos de Miedos en Niños, diseñado específicamente para esta investigación; las aplicaciones se hicieron de forma grupal, en los salones de clase. Los resultados muestran que los tipos de miedo que más tienen a lo largo de la infancia corresponden a los planteados dentro de la Teoría de la Paz o Equilibrio de Valdez Medina (2009). Por otro lado, se corroboró que tanto a nivel biológico, como sociocultural, las mujeres dijeron tener más miedos que los varones. Así mismo, se tuvo una tendencia en los resultados, en la cual se observa que a mayor edad, las personas tienden a reportar menor cantidad de miedos.

Descriptores: Miedo, niños, primaria, equilibrio, temores.

\section{ABSTRACT}

Aiming to identify the main types of fears that are present in the children of primary school, 300 participants from the city of Toluca were evaluated; they belonged to fifth and sixth grades, and were distributed evenly by sex. To obtain the required information, the instrument "Types of Fear in Children", designed specifically for this research was employed. The instrument was applied within the group, inside the classroom. The results show that the most frequent types of fears during childhood correspond with the ones considered in the Theory of the Peace or Balance of Valdez Medina (2009). On the other hand, there was corroborated from the biological as well as from the social and cultural levels, that women expressed to have more fears than men. Likewise, there was a tendency in the results that shows that as people grow old, they tend to have fewer fears.

Key words: Fear, children, primary school, balance, dreads.

\footnotetext{
* Para correspondencia a José Luis Valdez Medina, Arely Marlen Álvarez González, Diana González Gómez Tagle, Norma Ivonne González Arratia López Fuentes, Sergio González Escobar, dirigirse a la Facultad de Ciencias de la Conducta, UAEM. Av. Filiberto Gómez s/n, Col. Guadalupe Toluca México. Tel: (01722) 2-72-00-76, Fax: (01722)2-72-15-18, correos electrónicos: ochocedros@live.com.mx; arelymarlen_ag@hotmail.com; dana_2429@ yahoo.com.mx; nigalf@yahoo.com.mx; sergioglz4@hotmail.com, respectivamente.
} 


\section{INTRODUCCIÓN}

Uno de los temas más importantes que se han abordado como parte del estudio de la psicología, ha sido el de las emociones. Al respecto, se ha escrito que las emociones se constituyen como el principal sistema motivacional, determinado y organizado de la conducta de los humanos, y que pueden tener efectos favorables o desfavorables en la vida de cada uno de ellos (Garrido, 2006).

Así, hay autores (Manoni, 1984; Ostrosky, 2000) que indican que hay emociones agradables, como la alegría, el orgullo, la felicidad y el amor; y otras, que se catalogan como desagradables, entre las que se encuentran el dolor, la vergüenza, el miedo, el descontento, la culpabilidad, la cólera y la tristeza.

De estas, se ha encontrado que el miedo es un mecanismo de defensa básico, que a pesar de ser muy complejo, está compuesto fundamentalmente por un sentimiento emocional y una serie de cambios corporales (Whitehead, 1994), entre los que destacan la pilo-erección, el aumento de los latidos cardíacos, el ensanchamiento de las fosas nasales, la aceleración en la respiración, la sudoración, y la preparación para la acción requerida ante cualquier situación de amenaza o peligro inminente (Manoni, 1984; Ostrosky, 2000).

En este sentido, se ha propuesto que el miedo es la emoción central del comportamiento que se manifiesta como una constante de vida, ya que se presenta de manera invariable como parte del repertorio conductual instintivo y aprendido de las más diversas especies. Constituye un claro producto de la evolución biológica y psicosociocultural, ya que cualquier especie o miembro de la misma que fuera incapaz de experimentar miedo ante la presencia de alguna amenaza o peligro inminente, correría el riesgo de no reaccionar a tiempo y extinguirse o morir con más facilidad que otros seres que sí pudieran detectar las situaciones de peligro que les rodean y que producen algún nivel y tipo de miedo (Sassaroli \& Lorenzini, 2000; Valdez, 2009).

Por ello, se ha llegado a establecer que el miedo se constituye como el principal sistema de seguridad, motivación y búsqueda de adaptación a las nuevas circunstancias de vida que tienen los seres vivos, ya que en gran medida determina y organiza la conducta que éstos llegan a tener ante la presencia de algún tipo de amenaza o peligro. Esto es debido a que el miedo cumple con una función adaptativa de importancia trascendental para lograr la sobrevivencia y permanencia de todas y cada una de las especies que permanecen vivas en el planeta (Lutz, 1988; Méndez, 1999; Sánchez, 2006; Valdez, 2009).

Con base en lo anterior, es importante darse cuenta que de acuerdo con las premisas establecidas dentro de la Teoría de la paz o equilibrio (Valdez, 2009), puede decirse que el miedo tiene niveles y que existen distintos tipos de miedo; por ejemplo, la muerte o enfermedad; la soledad, abandono o rechazo; el castigo o venganza; las carencias o necesidades; la pérdida de libertad. Además el miedo cumple con dos funciones importantes. La primera consiste en actuar como señal de alarma que indica al organismo que hay algo que le amenaza, que le alteró el equilibrio o la estabilidad en la que se encontraba. La segunda es que el miedo funciona como una señal que informa al organismo que es necesario actuar o hacer algo, sea para encontrar y adaptarse a una nueva situación de estabilidad o de auto-organización, en donde se tenga el menor desgaste, es decir, que guía al organismo para tratar de ubicarse y adaptarse dentro de una nueva situación de paz o equilibrio (Valdez, 2009).

Ahora bien, aunque el miedo es un evento de carácter universal, en algunos reportes que hablan acerca del desarrollo psicológico del ser humano (Craig, 2007; Mussen, Conger \& Kagan, 1980; Papalia \& Wendkos, 2005), se encuentra que hay eventos que generan esta y otras experiencias de vida, y que estas no siempre son las mismas para todas las personas. Esto se debe a que las necesidades o carencias que se tienen y los niveles y tipos de miedo que se asocian con estas, van variando fundamentalmente con base en el sexo y la etapa de vida en la que cada una de las personas se encuentre (Valdez, 2009).

De esta forma, en algunos estudios se ha encontrado que una de las etapas de vida en la que se registran una mayor cantidad de eventos que provocan miedo, es la infancia (King, Hamilton \& Ollendick, 1988). Lo anterior se debe a que cada una de las interpretaciones que los niños hacen de los fenómenos que les pueden suscitar algún nivel o tipo de miedo reflejan algo de la concepción infantil del mundo (Méndez, 1999), donde la falta de información y de experiencia es importante. 
Es decir, ante ese desconocimiento de las situaciones, los niños son más vulnerables a ellas y no pueden evitarlas tan fácilmente como en el caso de las personas con mayor edad (Valdez, 2009).

$Y$ en este sentido, es claro que a medida que el individuo va creciendo y desarrollándose, lo más común es que las habilidades cognitivas y la experiencia que adquiere de la vida, le permitan llegar a tener un mejor entendimiento del ambiente en el que se desenvuelve. En consecuencia, hay un descenso en la cantidad y en el impacto que los miedos pueden llegar a tener en la persona (Mannoni, 1984), lo cual, al menos de forma hipotética, le permitiría tener una vida cada vez menos llena de miedos, que por supuesto, no siempre se cumple.

Por ello es que los miedos en la infancia tienden a ser muy frecuentes, de modo que prácticamente todos los niños refieren al menos un temor importante (King, Hamilton \& Ollendick, 1988), que no se experimenta con la misma intensidad, dependiendo de si es hombre o mujer (King \& Ollendick, 1997). Una de las explicaciones a lo anterior podría hacerse desde una perspectiva biológica que consiste en que, entre los mamíferos superiores (a los cuales pertenece el ser humano), los machos son por lo general de mayor tamaño y peso que las hembras, lo cual les permite tener las características físicas requeridas para poder enfrentarse a las adversidades o situaciones de miedo de manera más eficaz y exitosa que las hembras que, al no tener la misma fortaleza física que los machos, no pueden hacer frente a las amenazas con la misma facilidad (Méndez, 1999; Valdez, Díaz Loving \& Pérez, 2006).

Otra perspectiva que pudiera explicarlo es la social, que menciona Méndez (1999). Desde este punto de vista, hay un hallazgo global en nuestra cultura según el cual las niñas puntúan más alto en las pruebas de miedos que los niños, debido a que los padres educan a los hijos a reprimir sus emociones y presentarse ante la sociedad más valientes, mientras que a las niñas se les enseña a vivir protegidas por el sexo masculino. Sin embargo, es importante señalar que el dato se repite tanto en comunidades autónomas como en países desarrollados; lo cual concuerda con los resultados que se obtuvieron en la presente investigación, donde efectivamente el mayor grado de miedo lo obtuvieron las niñas.
Con base en lo anterior, el objetivo de la presente investigación fue el de poder detectar cuáles son los tipos de miedo más frecuentes en niños y niñas de quinto y sexto de primaria.

\section{MÉTODO}

\section{Participantes}

Se trabajó con una muestra no probabilística de tipo propositivo, compuesta por 300 alumnos de primaria de la ciudad de Toluca, clasificándolos de acuerdo con el grado escolar que cursan (quinto y sexto grado) con un promedio de edad de 11 años y repartidos equitativamente por sexo.

\section{Instrumento}

Se trabajó con un instrumento construido específicamente para el presente trabajo de investigación.

La construcción del instrumento tuvo como base la etno-metodología que requiere de la participación de las personas para llevar a cabo la obtención de los reactivos (Valdez Medina, 1994). Se incluyen como reactivos del instrumento las respuestas provenientes de una muestra de participantes con las mismas características de edad, sexo, escolaridad, etc., que tienen aquellos a los que se pretende evaluar con el instrumento una vez construido. De esta manera, los reactivos que lo conforman fueron obtenidos mediante la aplicación previa de la técnica de redes semánticas naturales en niños y niñas de la misma edad, escolaridad y sexo, los cuales fueron incluidos como parte de la muestra seleccionada para participar en esta investigación. Para la primera fase (de la que no se reportan los resultados), se utilizó el siguiente reactivo: “¿Cuáles son los principales miedos o temores que has tenido a lo largo de lo que llevas de vida?"

Con base en los términos obtenidos, se construyó, validó y se hizo confiable el instrumento denominado Los miedos de los niños, el cual consta de 60 reactivos con una escala tipo Likert de cinco opciones de respuesta, donde el número uno (1), indica que el niño o niña nunca le ha tenido miedo a eso, y donde la opción marcada con el número cinco (5), muestra que siempre le ha tenido mucho miedo a eso. Los datos psicométricos obtenidos dejan ver que el instrumento permite explicar el $36.83 \%$ de la varianza, con una consistencia 
interna de Alpha de Cronbach $=0.933$, lo cual habla de un instrumento con buenos índices de confiabilidad y validez.

\section{Procedimiento}

Las aplicaciones tanto en la primera como en la segunda fase se hicieron de forma grupal dentro de los salones de clase.

\section{RESULTADOS}

De acuerdo con los resultados obtenidos en el análisis factorial con rotación VARIMAX que se obtuvo para ambos grupos, se encontraron 17 factores con valores Eigen mayores a uno. Sin embargo, se eligieron solamente los primeros cinco con base en la claridad conceptual y quiebre de la varianza (Valdez Medina, 1994). Estos factores permiten explicar el $36.83 \%$ de la varianza, con una consistencia interna de Alpha de Cronbach $=0.933$.

De esta forma, se encontró que para los niños, los miedos más frecuentes conllevan situaciones de rechazo, abandono familiar, posibilidad de morir, castigo o agresión física y por último la falta de libertad (tabla 1).

Tabla 1. Análisis factorial de los miedos más recurrentes en alumnos de $5^{\circ}$ y $6^{\circ}$ grado de primaria.

\begin{tabular}{|c|c|c|c|c|c|}
\hline Reactivos & Rechazo & $\begin{array}{l}\text { Abandono } \\
\text { familiar }\end{array}$ & $\begin{array}{l}\text { Posibilidad } \\
\text { de morir }\end{array}$ & $\begin{array}{c}\text { Castigo o } \\
\text { agresión física }\end{array}$ & $\begin{array}{l}\text { Falta de } \\
\text { libertad }\end{array}$ \\
\hline Que se burlen de mi & ,640 & & & & \\
\hline Ser feo & ,618 & & & & \\
\hline Que me discriminen &, 604 & & & & \\
\hline No tener amor &, 562 & & & & \\
\hline Que me agredan &, 517 & & & & \\
\hline Carencias materiales & ,479 & & & & \\
\hline Que sea rechazado & ,445 & & & & \\
\hline Que me dejen mis padres & & ,750 & & & \\
\hline Que se muera mi familia & &, 687 & & & \\
\hline Que no me quieran mis padres & & ,602 & & & \\
\hline Los accidentes & &, 501 & & & \\
\hline Que se peleen mis padres & & ,437 & & & \\
\hline Los extraños & & & ,687 & & \\
\hline Los roba-chicos & & &, 672 & & \\
\hline Las alturas & & &, 572 & & \\
\hline Que se venguen de mi & & &, 521 & & \\
\hline Perderme & & &, 504 & & \\
\hline Los castigos & & & &, 605 & \\
\hline Depender de alguien & & & &, 547 & \\
\hline Mis papás enojados & & & &, 517 & \\
\hline
\end{tabular}




\begin{tabular}{|l|l|c|c|c|c|}
\hline \multicolumn{1}{|c|}{ Reactivos } & Rechazo & $\begin{array}{c}\text { Abandono } \\
\text { familiar }\end{array}$ & $\begin{array}{c}\text { Posibilidad } \\
\text { de morir }\end{array}$ & $\begin{array}{c}\text { Castigo o } \\
\text { agresión física }\end{array}$ & $\begin{array}{c}\text { Falta de } \\
\text { libertad }\end{array}$ \\
\hline Que me golpeen & & & &, 449 &, 608 \\
\hline Tener obstáculos & & & & &, 595 \\
\hline No tener libre elección & & & & &, 515 \\
\hline No seguir estudiando & 12.65 & 3.36 & 2.19 & 2.03 & 1.86 \\
\hline Que me obliguen a hacer algo & & & & & \\
\hline VALORES EIGEN & 36.83 & & & & \\
\hline VARIANZA TOTAL & .933 & & & & \\
\hline ALPHA TOTAL & & & & & \\
\hline
\end{tabular}

Posteriormente con la finalidad de conocer las particularidades de las respuestas de los Alumnos de $5^{\circ} \mathrm{y}$ $6^{\circ}$ grado de primaria, se realizó una comparación por reactivo y por grado Mediante el uso de la prueba $\mathrm{T}$ de Student, se observó que los alumnos de $5^{\circ}$ grado tienen más miedo a: la muerte (a ser hospitalizados, a la oscuridad, a las películas de terror, las pesadillas, a las enfermedades, al dolor y a los accidentes); la soledad, el abandono o el rechazo (a ser rechazados, a ser feo(a), al rompimiento de relaciones, a la soledad, a perderse, a equivocarse, a que se muera su familia, a que los dejen sus padres y a que los discriminen); a las carencias $o$ necesidades (pelearse con sus padres, a no tener hijos, a que se enojen sus padres, a no seguir estudiando, a que no los quieran sus padres, a no casarse, a no tener amor, a sufrir carencias materiales y a que se peleen sus padres); al castigo o venganza (que se burlen de ellos, que los regañen, que los golpeen, a las personas que mandan, a los castigos, a que los agredan y a ser expulsados); y por último a la perdida de la libertad (a ser secuestrados, a depender de alguien, a que los obliguen a hacer algo, a no tener libre elección, a no poder jugar, a las cosas nuevas en su vida, a la falta de libertad, a no poder expresarse y a ser esclavos).

Respecto a los alumnos de $6^{\circ}$ grado se encontró que aparentemente por el puntaje obtenido, ellos no presentan el mismo temor en comparación con los alumnos de $5^{\circ}$ grado, en la mayoría de los reactivos presen- taron un más alto nivel de miedo. Por lo tanto se considera que los alumnos de $5^{\circ}$ grado son más miedosos que los alumnos de $6^{\circ}$ grado (tabla 2 ).

De igual manera se realizó la comparación por sexo para los dos grados en estudio con lo que se determinó que las mujeres presentan mayor miedo que los hombres. Ello se observa en la siguiente tabla 3, donde se muestra que las mujeres tienen mayor número de miedos relacionados con la categoría de miedo o enfermedad (la oscuridad, los payasos, al dolor, los accidentes, a los extraños y a las alturas); con la soledad, abandono o rechazo (perderse, al silencio, a equivocarse y a ser esclava); con las carencias y necesidades (que se peleen sus padres y perder dinero); con el castigo o venganza (que las regañen y las golpeen); con la pérdida de la libertad (a un secuestro, a los roba-chicos y la falta de libertad). En contraste con las mujeres, los hombres tienen más miedos relacionados con la soledad, abandono o rechazo (romper relaciones) y con la pérdida de la libertad (a ir a la escuela) (tabla 3). 
Tabla 2. Miedos más frecuentes en alumnos de $5^{\circ}$ y $6^{\circ}$ grado de primaria: una comparación por reactivo y por grado

\begin{tabular}{|c|c|c|c|c|c|c|c|}
\hline \multirow[b]{2}{*}{ Tipos de miedos } & \multirow[b]{2}{*}{ Reactivo } & \multirow[b]{2}{*}{$\mathbf{T}$} & \multirow[b]{2}{*}{$\mathbf{P}$} & \multicolumn{2}{|c|}{ QUINTO } & \multicolumn{2}{|c|}{ SEXTO } \\
\hline & & & & $\bar{X} 1$ & $\sigma 1$ & $\bar{X} 2$ & $\sigma 2$ \\
\hline \multirow{7}{*}{ Muerte o enfermedad } & Ser hospitalizado(a) & 3.44 & .001 & 2.56 & 1.26 & 2.04 & 1.35 \\
\hline & La oscuridad & 4.74 & .000 & 2.33 & 1.44 & 1.64 & 1.03 \\
\hline & Las películas de terror & 3.33 & .001 & 2.19 & 1.26 & 1.74 & 1.05 \\
\hline & Las pesadillas & 2.44 & .015 & 2.85 & 1.40 & 2.44 & 1.22 \\
\hline & Las enfermedades & 2.20 & .028 & 2.82 & 1.39 & 2.48 & 1.27 \\
\hline & El dolor & 2.37 & .018 & 2.98 & 1.39 & 2.62 & 1.27 \\
\hline & Los accidentes & 2.48 & .014 & 3.75 & 1.42 & 3.33 & 1.50 \\
\hline \multirow{8}{*}{ Soledad, abandono, rechazo } & Ser rechazado(a) & 5.51 & .000 & 2.67 & 1.39 & 1.84 & 1.19 \\
\hline & Ser feo (a) & 2.92 & .004 & 1.92 & 1.40 & 1.50 & 1.06 \\
\hline & Los rompimientos de relaciones & 3.44 & .001 & 2.66 & 1.59 & 2.08 & 1.34 \\
\hline & La soledad & 4.61 & .000 & 3.06 & 1.55 & 2.26 & 1.42 \\
\hline & Perderse & 2.63 & .009 & 3.42 & 1.49 & 2.94 & 1.61 \\
\hline & Que se muera su familia & 2.41 & .016 & 4.51 & 0.98 & 4.18 & 1.33 \\
\hline & Equivocarse & 2.54 & .011 & 2.78 & 1.42 & 2.38 & 1.34 \\
\hline & Ser discriminado(a) & 6.17 & .000 & 3.14 & 1.51 & 2.12 & 1.34 \\
\hline \multirow{11}{*}{ Carencias o necesidades } & Pelearse con sus padres & 6.65 & .000 & 3.16 & 1.45 & 2.12 & 1.24 \\
\hline & Que se peleen sus padres & 4.67 & .000 & 3.66 & 1.36 & 2.90 & 1.46 \\
\hline & No tener hijos & 4.18 & .000 & 2.76 & 1.59 & 2.03 & 1.41 \\
\hline & Las personas que mandan & 3.17 & .002 & 2.00 & 1.30 & 1.57 & 1.03 \\
\hline & Abandono de los padres & 2.43 & .015 & 3.96 & 1.33 & 3.56 & 1.54 \\
\hline & Sus papás enojados & 4.07 & .000 & 3.12 & 1.31 & 2.49 & 1.35 \\
\hline & No seguir estudiando & 4.69 & .000 & 3.33 & 1.59 & 2.48 & 1.48 \\
\hline & No ser querido(a) por los padres & 4.66 & .000 & 3.78 & 1.44 & 2.96 & 1.57 \\
\hline & No casarse & 4.09 & .000 & 2.24 & 1.36 & 1.64 & 1.13 \\
\hline & No tener amor & 4.20 & .000 & 2.91 & 1.53 & 2.22 & 1.31 \\
\hline & Carencias materiales & 4.41 & .000 & 2.64 & 1.41 & 1.96 & 1.24 \\
\hline \multirow{6}{*}{ Castigo o venganza } & Ser regañado(a) & 2.32 & .021 & 2.24 & 1.14 & 1.95 & 1.03 \\
\hline & Ser golpeado(a) & 4.06 & .000 & 2.97 & 1.39 & 2.32 & 1.38 \\
\hline & Los castigos & 2.46 & .014 & 2.30 & 1.28 & 1.96 & 1.08 \\
\hline & Ser objeto de burlas & 2.96 & .003 & 2.50 & 1.39 & 2.04 & 1.29 \\
\hline & Ser agredido(a) & 4.69 & .000 & 3.01 & 1.40 & 2.28 & 1.27 \\
\hline & Ser expulsado(a) & 3.73 & .000 & 3.72 & 1.41 & 3.08 & 1.52 \\
\hline \multirow{9}{*}{ Pérdida de la libertad } & Depender de alguien & 4.18 & .000 & 2.27 & 1.47 & 1.64 & 1.09 \\
\hline & Ser obligado a hacer algo & 4.36 & .000 & 2.85 & 1.51 & 2.15 & 1.24 \\
\hline & No tener libre elección & 3.38 & .001 & 2.98 & 1.49 & 2.40 & 1.51 \\
\hline & No poder jugar & 3.45 & .001 & 1.86 & 1.20 & 1.43 & 0.90 \\
\hline & Las cosas nuevas de su vida & 2.29 & .022 & 1.94 & 1.27 & 1.62 & 1.08 \\
\hline & La falta de libertad & 3.47 & .001 & 2.70 & 1.44 & 2.14 & 1.34 \\
\hline & No poder expresarse & 4.00 & .000 & 2.45 & 1.28 & 1.88 & 1.16 \\
\hline & Tener obstáculos & 3.84 & .000 & 2.34 & 1.30 & 1.80 & 1.07 \\
\hline & Ser esclavo(a) & 3.61 & .000 & 3.59 & 1.49 & 2.96 & 1.54 \\
\hline
\end{tabular}


Tabla 3. Miedos más frecuentes en alumnos de $5^{\circ}$ y $6^{\circ}$ grado de primaria: una comparación por reactivo y por sexo

\begin{tabular}{|c|c|c|c|c|c|c|c|}
\hline \multirow{2}{*}{ Tipos de miedos } & \multirow{2}{*}{ Reactivo } & \multirow{2}{*}{$\mathrm{t}$} & \multirow{2}{*}{$\mathrm{p}$} & \multicolumn{2}{|c|}{ HOMBRES } & \multicolumn{2}{|c|}{ MUJERES } \\
\hline & & & & $x 1$ & DE1 & $\times 2$ & DE2 \\
\hline \multirow{6}{*}{ Muerte o enfermedad } & La oscuridad & 2.0 & .045 & 1.84 & 1.26 & 2.14 & 1.31 \\
\hline & Los payasos & 2.05 & .041 & 1.64 & 1.24 & 1.96 & 1.39 \\
\hline & El dolor & 2.0 & .044 & 2.64 & 1.26 & 2.96 & 1.40 \\
\hline & Los accidentes & 2.5 & .011 & 3.32 & 1.50 & 3.76 & 1.41 \\
\hline & Los extraños & 2.7 & .006 & 2.56 & 1.45 & 3.02 & 1.43 \\
\hline & Las alturas & 3.8 & .000 & 2.57 & 1.68 & 3.30 & 1.59 \\
\hline \multirow{5}{*}{ Soledad, abandono o rechazo } & Los rompimientos de relaciones & 2.0 & .045 & 2.54 & 1.59 & 2.20 & 1.39 \\
\hline & Perderse & 4.6 & .000 & 2.77 & 1.58 & 3.59 & 1.45 \\
\hline & El silencio & 2.9 & .004 & 1.89 & 1.28 & 2.36 & 1.48 \\
\hline & Equivocarse & 3.3 & .001 & 2.32 & 1.34 & 2.84 & 2.40 \\
\hline & Ser esclavo (a) & 2.0 & .040 & 3.09 & 1.55 & 3.46 & 1.53 \\
\hline \multirow{2}{*}{ Carencias y necesidades } & Que se peleen sus padres & 3.0 & .002 & 3.02 & 1.46 & 3.54 & 1.42 \\
\hline & Perder dinero & 2.4 & .017 & 2.28 & 1.41 & 2.66 & 1.37 \\
\hline \multirow{2}{*}{ Castigo o venganza } & Que me regañen & 2.33 & .021 & 1.95 & 1.11 & 2.24 & 1.06 \\
\hline & Que me golpeen & 4.06 & .000 & 2.32 & 1.33 & 2.97 & 1.45 \\
\hline \multirow{4}{*}{ Perdida de la libertad } & Un secuestro & 3.3 & .001 & 3.9 & 1.46 & 4.41 & 1.18 \\
\hline & Ir a la escuela & 3.3 & .001 & 1.42 & 0.97 & 1.12 & 0.48 \\
\hline & Los roba-chicos & 3.1 & .002 & 2.87 & 1.59 & 3.42 & 1.43 \\
\hline & Falta de libertad & 2.04 & .042 & 2.25 & 1.35 & 2.58 & 1.47 \\
\hline
\end{tabular}

\section{DISCUSIÓN}

De acuerdo con los resultados obtenidos, se observa que todos los niños han experimentado miedo en alguna ocasión. Al respecto, Méndez (1999) menciona que los niños experimentan miedos a lo largo de su desarrollo y que la mayoría son pasajeros, de poca intensidad y propios de una edad determinada.

Valiente (2003) señala que los miedos son más comunes durante la infancia, presentan un curso evolutivo y su prevalencia va en función de la edad. Es importante decir que con base en que todos los seres vivos nos movemos entre el nacimiento y la muerte, el mayor y único miedo que tenemos es el de morir, y este a su vez tiene una íntima relación con otros tipos de miedo que nos pueden acercar a la experiencia de muerte, como son la soledad, el abandono o rechazo; las carencias o necesidades; el castigo o venganza y la pérdida de la libertad (Valdez, 2009). De acuerdo con los resultados obtenidos, se observó que los niños de $5^{\circ}$ y $6^{\circ}$ grado de primaria tienen mayor miedo a situaciones relacionadas con el rechazo, el abandono familiar, la posibilidad de morir, el castigo o agresión física y la falta de libertad. Con respecto al abandono, Pelechano (1984) alude que probablemente algunos niños han vivido separaciones forzosas, como la muerte de un ser querido, el divorcio de los padres o la hospitalización por alguna enfermedad grave, que les ha trastornado profundamente. A 
raíz de estos sucesos, son más vulnerables a reaccionar ante separaciones cotidianas, ya que ellos sienten que son causantes principales de dicho acontecimiento.

Sin embargo, no todos los miedos típicos de una edad aparecen al mismo tiempo, sino que surgen, desaparecen y cambian a medida que el individuo crece y se desarrolla (Valdez, 2009). Hay un proceso predecible en la aparición y extinción de los miedos normales de una edad, aunque puede haber algunos que se mantengan a lo largo de la vida por no haber sido resueltos a tiempo. Sin embargo, los miedos emergen, se estabilizan y declinan (Marina, 2006).

En relación con los resultados que se obtuvieron en el análisis por grado se puede observar que los alumnos de $6^{\circ}$ grado no tuvieron la misma intensidad de miedo que los alumnos de $5^{\circ}$ grado, debido a que los alumnos llevan un año de diferencia en cuanto a la edad, lo cual es significativo en la infancia.

A lo largo de la existencia individual, el miedo cambiará de contenido y de grado, sin desaparecer nunca por completo, pues cada edad tiene sus miedos específicos y pesadillas particulares, por lo que ninguna etapa de la vida humana queda libre de alguno de los tipos de miedo (Mannoni, 1984).

Según estudios de Ramón y Cajal (2009), se pueden observar diferencias importantes en los primeros años de vida, ya que los niños presentan miedo a que los regañen, que los golpeen, al dolor, a los accidentes o al rompimiento de relaciones, entre otros. Es decir, con el paso de los años los temores infantiles evolucionan y van cambiando de miedos físicos (ser feo (a), que me agredan) a miedos sociales como por ejemplo ser rechazado (a), también es importante mencionar que, hay algunos miedos que perduran a lo largo de toda la vida del ser humano.

En relación con la diferencia de miedos que existe entre los sexos, es necesario destacar que biológicamente los hombres y las mujeres presentan características físicas distintas. Así, se propone que los niños tienden a ser constitucionalmente más fuertes y por tanto estar mejor dotados para la defensa y ataque que las niñas, gracias a lo cual muestran menos conductas temerosas (Darwin, 1871). Asimismo, los niños poseen más fortaleza, más habilidades motoras, mayor percepción o agudeza visual propios para detectar de lejos las amenazas y así poder evitar con mayor facilidad los peligros que se les presentan (Valdez, 2006). Lo anterior coincide con lo dicho por Mira y López (1957) acerca de que los niños temen a la ruptura de relaciones por su invalidez o ineficiencia para satisfacer sus necesidades, ya que las niñas le temen a la agresión física porque se sienten incapaces para defenderse.

Otras explicaciones en relación con la mayor intensidad y frecuencia de los miedos en las niñas se refieren a la deseabilidad social y a las pautas educativas familiares. La primera hipótesis sugiere que los niños manifiestan menos miedos de los que sienten en realidad porque es lo que se espera de ellos. La segunda explicación se refiere a la educación que reciben las niñas, pues los padres suelen emplear con sus hijas estrategias que fomentan comportamientos temerosos y dependientes, frente a los niños a quienes primordialmente se les refuerza la independencia y la seguridad personal (Merrel y Gimpel, 1998).

Según Sassaroli y Lorenzini (2002) esto sería coherente con el rol general de comportamiento femenino (necesidad de protección, temerosidad, exteriorización de los sentimientos, etc.) frente al rol masculino (ausencia de temor, valor y defensa del sexo débil).

Otro aspecto relevante que menciona Méndez (1999), es que las niñas registran una puntuación más alta en las pruebas de miedos que los niños, y que este hallazgo es generalizado en la cultura mexicana. Sin embargo, es importante señalar que el dato se repite tanto en comunidades autónomas como en países desarrollados, lo cual concuerda con los resultados que se obtuvieron en la presente investigación, donde efectivamente el mayor grado de miedo lo obtuvieron las niñas.

En conclusión, todo parece indicar que el miedo es el motor central de la conducta humana que surge ante un estimulo sorpresivo y que, de no resolverse a tiempo, se manifiesta con ansiedad (Heiliger, 1988). Se considera que la paz o equilibrio es la tendencia natural de la vida, lo que de forma directa o indirecta pretendemos alcanzar mediante la aplicación de nuestras tendencias de personalidad natural o individual que cubren nuestras necesidades o carencias y que nos permiten evitar cualquier contacto con los niveles, tipos, reacciones y formas del miedo. De esta forma se puede comprobar que los miedos presentados por los niños se pueden clasificar dentro de los que propone la 
teoría de la paz o el equilibrio (Valdez, 2009), que son: muerte o enfermedad, soledad, abandono o rechazo, carencia o necesidad, castigo o venganza y pérdida de la libertad; y que las diferencias encontradas por sexo, dejan ver que a pesar del peso de la cultura, la base biológica sigue influyendo de forma importante en el desarrollo de nuestra conducta y en la construcción y desarrollo de las tendencias de personalidad.

\section{REFERENCIAS}

Craig, G. (2007). Desarrollo Psicológico. México: Prentice Hall Hispanoamérica.

Darwin, C. (1871). On the origin of species. London: UK Murray.

Garrido, L. (2006). Apego, emoción y regulación emocional: Implicaciones en la salud. Revista Latinoamericana de Psicología, 38 (3), 493-507. Disponible: http:// redalyc.uaemex.mx/.

Heiliger, A. (1988). La angustia y el miedo en el niño. México: Fondo de Cultura.

King, N. J., Hamilton, D. I. \& Ollendick, T. H. (1988). Children's phobias: A behavioral perspective. Chichester: Wiley.

King, N. J. \& Ollendick, T. H. (1997). Annotation: Treatment of childhood phobias. Journal Child Psychology and Psychiatry, 38, 389-499.

Lutz, C. (1988). Unnatural Emotions: everyday sentiments on a Micronesian atoll \& their challenge to western theory. Estados Unidos: Univerity of Chicago

Mannoni, P. (1984). El miedo. México: Fondo de cultura económica.

Marina, J. (2006). Anatomía del miedo: un tratado sobre la valentía. Barcelona: Anagrama.

Méndez, F. (1999). Miedos y temores en la infancia: ayudar a los niños a superarlos. Madrid: Pirámide.

Merrell, K. W. \& Gimpel, G. A. (1998). Social Skills of Children and Adolescents: Conceptualization, Assessment, Treatment (Hardcover). New Jersey London: Lawrence Erlbaum Associates.

Mira y Lopez (1957). Los cuatro gigantes del alma. México: Trillas.

Musen, P., Conger, J. \& Kagan, J. (1980). Desarrollo Psicológico en el niño. México: Trillas.

Ostrosky, F. (2000). ¡Toc,toc!, ¿Hay alguien ahí?. Mexico: Infored.

Papalia, E. D. \& Wendkos, D. S. (2005). Psicologia del desarrollo. Mexico: McGraw Hill.
Pelechano, V. (1984). Programas de intervención psicológica en la infancia: miedos. Análisis y modificación de conducta. Valencia: Alfaplús.

Ramón y Cajal (2009). Miedos y temores en la infancia. Disponible en http://www.rcajal.es/Proyeto/educarHoy/2008-10\%20LosMiedosInfantiles.aspx.

Sánchez, G. (2006). Los miedos infantiles en la literatura para niños. Salamanca: Graficas LOPE.

Sassaroli. S \& Lorenzini. R. 2000. Miedos y fobias: causas, características y terapias. España: Paidós.

Valdez, J. (1994). El auto concepto del mexicano: estudios de validación. Tesis de Doctorado. Facultad de Psicología. Universidad Nacional Autónoma de México.

Valdez, J. (2009). Teoría de la paz o equilibrio. México: Edamex.

Valdez, J., Díaz Loving, R. \& Perez, B. R. (2006). Los hombres y las mujeres de México: Dos mundos distantes y complementarios. Mexico: UAEM.

Valiente, R. (2003). Diferencias según la edad en la prevalencia e intensidad de los miedos durante la infancia y la adolescencia. Datos basados en el FSSC-R.

Whitehead, T. (1994). Miedos y Fobias: cómo superarlos. España: Trikal.
Fecha de recepción: diciembre 2009 Fecha de publicación: mayo 2010 